\title{
Der Beitrag der Steiermark zum Marine Littering
}

\author{
Dominik Huter · Roland Pomberger
}

Online publiziert: 10 . August 2020

(C) Der/die Autor(en) 2020

Zusammenfassung Da sich immer mehr Kunststoffe im Meer ansammeln, wurde diese Studie für das Land Steiermark erstellt, um mittels Materialflussanalyse die Emissionen im Land, als auch den Transfer über Flüsse bis ins Meer abzuschätzen. Die Parameter für das Modell entstammen der Literatur und basieren teilweise auch auf begründeten Annahmen. Littering ist mit $45 \%$ der modellierten KunststoffEmissionen die größte Emissionsquelle, gefolgt vom Abrieb vom Verkehr (Reifen $36 \%$, Fahrbahnmarkierung $2 \%$ ), sowie von Schuhsohlen (4\%) und Kunstrasen und anderen Sportflächen (6\%), welche alle diffuse Quellen bilden. Der Abrieb synthetischer Fasern von Wäsche (3\%), Innenraumstäube (2\%) und Microbeads $(<1 \%)$, in Pflegeprodukten, stellen bedeutende punktuelle Einträge über die Abwässer der Haushalte in die Kanalisation und weiter in die Kläranlagen. Etwa $3 \%$ des gesamten Mikroplastiks gelangen über Klärschlämme und Komposte in die Umwelt. Die modellierten Kunststoff-Emissionen der Steiermark werden nach den Szenarien dieser Studie insgesamt auf 1545-5353 Tonnen pro Jahr (t/a) geschätzt. Die Kunststoff-Emissionen gelangen hauptsächlich über die Oberflächengewässer (427-1179t/a) und anschließend über die Donau bis ins Schwarze Meer (43-109t/a). Im Vergleich zu den Konzentrationen in Flüssen und anderen Modellen sind die im Transfermodell quantifizierten Emissionen in Oberflächengewässer und das Meer relativ hoch. Die Steiermark emittiert als Binnenland in etwa $0,0001 \%$ des globalen Kunststoffes, welcher pro Jahr vom Land ins Meer gelangt, und um einen Faktor von in etwa 16 (7-48) weniger

D. Huter, BSc. BA. $(\bowtie)$.

Univ.-Prof. DI Dr. R. Pomberger

Lehrstuhl für

Abfallverwertungstechnik

und Abfallwirtschaft (AVAW),

Montanuniversität Leoben,

Franz-Josef-Straße 18, 8700 Leoben,

Österreich

dominik.huter@gmx.at
Kunststoffe ins Meer als ein Mensch der Erde im Durchschnitt.

\section{Schlüsselwörter}

Kunststoffemissionen - STAN · Materialflussanalyse - Steiermark . Mikroplastik · Flüsse · Böden · Verkehr · Transfer · Autoreifen . Kunstrasen · Wäsche · Kompost . Klärschlamm · Kläranlage · Abwasser · Mur · Schwarzes Meer

\section{The contribution to marine littering of the province of Styria, Austria}

Abstract As more plastics are accumulating in the sea this study was created for the province of Styria to use material flow analysis to estimate the emissions in the country as well as the transfer via rivers into the sea. The parameters for the model come from the literature and are partly based on well-founded assumptions. Littering is the largest emission source with $45 \%$ of the modelled plastic emissions, followed by abrasion caused by traffic (tires $36 \%$, lane marking $2 \%$ ), as well as shoe soles $(4 \%)$ and artificial turfs and other sports surfaces $(6 \%)$, all of which are diffuse sources. The abrasion of synthetic fibers from laundry (3\%), interior dusts (2\%) and microbeads in care products $(<1 \%)$ represent significant entries via the household waste water into the sewage system. Microplastics are transported to the environment, via sewage sludge or compost from biogenic municipal waste (3\%). The modelled plastic emissions of Styria are estimated, according to the scenarios of this study, in total at $1545-5353$ tons per year (t/a). Plastic emissions mainly come from surface waters (427-1179t/a) and then across the Danube to the Black Sea (43-109t/a). Compared to the concentrations in rivers and other models, the emissions into surface waters and the sea quantified in the transfer model are relatively high. As a landlocked country, Styria emits approximately $0.0001 \%$ of the global plastic that enters the sea from land each year and a factor of approximately 16 (7-48) fewer plastics than the average person on earth.

Keywords Marine Litter · Plastic emissions $\cdot$ STAN $\cdot$ Material flow analysis · Styria · Microplastics · Rivers . Soils - Traffic - Transfer - Car tyres · Artificial turf - Laundry - Compost . Sewage sludge $\cdot$ Sewage treatment plant $\cdot$ Wastewater $\cdot$ Mur $\cdot$ Black Sea

\section{Problemstellung}

Die Verschmutzung der Meere durch Mikro- und Makroplastik wird seit den späten 1960er Jahren wissenschaftlich diskutiert. In einer der ersten Studien (Carpenter et al. 1972) wurde festgestellt, dass Kunststoffabfälle in den küstennahen Meeresgewässern von Großbritannien schwimmen und in kleinere Stücke zerfallen. Die erste systematische Analyse der geographischen Verbreitung von Mikroplastik-Partikeln in Flusssystemen behandelte dadurch entstandene Umwelteffekte, wie z.B. toxische Gefahren für Meereslebewesen, und die Frage der Persistenz von Kunststoffen (Colton et al. 1974). Später wurde die Masse des aufgenommenen Kunststoffs mit der Aufnahme von PCBs durch Seevögel korreliert (Ryan et al. 1988), was darauf hindeutet, dass Seevögel durch die Aufnahme von inertem Kunststoff zusätzlich toxische Chemikalien aufnehmen.

Eine Vielzahl umweltpersistenter Chemikalien, darunter auch einige intentionell $\mathrm{zu}$ Kunststoffen zugesetzte Stoffe (Additive), wie Phthalat-Weichmacher, sind schwach östrogen (Jobling et al. 1995) und beeinflussen daher das endokrine System von Organismen, indem sie das primäre weibliche $\mathrm{Ge}$ schlechtshormon imitieren. Die Oberfläche von Mikroplastik-Partikeln ist hydrophob und adsorbiert daher die genannten hydrophoben Schadstoffe, aber auch persistente organische Schadstoffe (Rios et al. 2007) aus dem Meerwasser. Mikroplastik-Partikel können durch Wind und Wasser an weit entfernte Orte transportiert werden (Teuten et al. 2007). Es wurde deutlich, 
dass diese Schadstoffe in der Umwelt, in der Nahrungsmittelkette und im Trinkwasser weit verbreitet und persistent sind (Turner und Sharpe 1997).

Thompson et al. (2004) prägten den Begriff "Mikroplastik“ in dem sie die globale räumliche Ausdehnung und Akkumulation dieser Art von Kontamination aufzeigten. Kurz danach dokumentierten erste Experimente die Akkumulation und Fragmentierung von Kunststoffabfällen und die Aufnahme von Mikroplastik-Partikeln in das Gewebe von Muscheln, wonach die Möglichkeit der Aufnahme in das Gewebe lediglich eine Frage der Partikelgröße ist (Browne et al. 2008). Die Intensivierung der Forschung zu Mikroplastik nahm ab dem Jahr 2010 ihren weiteren Verlauf (Connors et al. 2017).

Der Verbrauch verschiedener Kunststoffe ist in Österreich seit den 1960erJahren um etwa das Zehnfache gestiegen, was heute etwa die Menge von 875.000 t/a gesammeltem Kunststoff ausmacht (Liebmann 2015). Heute macht gesammelter Kunststoffabfall in Österreich etwa 875.000 t/a aus (Liebmann 2015). Inzwischen hat sich das Vorkommen von Mikroplastik-Partikeln, als eine relativ neu aufkommende Art von Kontaminanten, weltweit zum Thema für die Öffentlichkeit und staatliche Behörden entwickelt (Liu et al. 2018).

Da $70 \%$ des an europäischen Stränden gefundenen „Marine Litter" Einwegprodukte sind, kündigte das Europäische Parlament eine Strategie zur Bekämpfung der Verschmutzung durch Kunststoffe an, indem es die Verwendung einer Vielzahl von Einwegprodukten bis 2021 verbietet und die Verwendung von Kunststoffen, bei denen keine Alternativen verfügbar sind, bis 2025 um mindestens $25 \%$ zu reduzieren versucht (European Parliament 2018).

Alle Regionen der Erde tragen signifikant zu den Emissionen von Kunststoffen bei (Boucher und Friot 2017). $90 \%$ der Kunststoffe in den Weltmeeren stammen aus landbasierten Quellen, von Flüssen, Küstenstädten, Häfen, Kläranlagen und unkontrollierten, küstennahen Deponien (Andrady 2011). Es gibt einen engen Zusammenhang zwischen den gefundenen Kunststoffsorten in marinen Gewässern und jenen, welche durch die Emissionsquellen an Land freigesetzt werden (Jang et al. 2020).

Das Weltwirtschaftsforum hat prognostiziert, dass die weltweite Kunst- stoffproduktion von 2014 bis 2050 um den Faktor 3, von 311 auf 1124 Mio. $\mathrm{t} / \mathrm{a}$, steigen wird. Im prognostizierten „business-as-usual“-Szenario wird im Jahr 2050 die Masse an Kunststoffen im Meer die Masse der Fische in den Ozeanen überholt haben. Das heutige Verhältnis beträgt 1:5 (World Economic Forum 2016).

Die Kontamination mit Mikroplastik war in sämtlichen untersuchten Sedimenten aus Flussbetten in einer britischen Studie allgegenwärtig (Hurley et al. 2018). Die Konzentrationen von Mikroplastik in Flüssen korrelieren mit dem Durchflussvolumen und der Größe des Einzugsgebietes (Mani \& Burkhardt-Holm 2020; Siegfried et al. 2017). Das von den Flusssystemen exportierte Mikroplastik lässt sich im einfachsten Fall mit der Dichte der Bevölkerung und deren anteilsmäßigem Anschluss an Kläranlagen, dem Pro-Kopf-Eintrag an Mikroplastik und der KläranlagenEffizienz modellieren. Weiters spielen für die exportierte Menge an Mikroplastik Überflutungen eine Rolle (Mani \& Burkhardt-Holm 2020; Siegfried et al. 2017).

In einer Studie zur Kunststoffbelastung des Schwarzen Meeres (Slobodnik et al. 2018) wurden die Immissionsanteile durch Flüsse ins Schwarze Meer zusammengefasst: $20 \%$ (Masse) Trinkflaschen, $14 \%$ Verpackungen, $14 \%$ Bruchstücke (Mikroplastik), $11 \%$ Tragetaschen und $9 \%$ Food-Container, während im offenen Gewässer viel mehr Bruchstücke $(68 \%)$ gefunden wurden. Das korreliert mit den Ergebnissen von Sun et al. (2019), die sekundäres Mikroplastik, entstanden aus der Degradation größerer Teile, als dominanten Typus in Gewässern feststellen (Sun et al. 2019). Laut Slobodnik et al. (2018) bestehen bereits $83 \%$ des Marine Litter im Schwarzen Meer aus Plastik. Dichte Objekte können ebenso den Meeresboden erreichen. Slobodnik et al. (2018) bewerten den Eintrag von Kunststoffen über Flüsse in das Schwarze Meer als eine der Hauptquellen und finden vor allem im Nordwesten (Donaumündung) hohe Konzentrationen. Die AutorInnen schätzen, dass die Donau allein ca. $80 \%$ der jährlichen Kunststoffeinträge durch Flüsse ins Schwarze Meer mit sich bringt. In Sedimenten der Tiefsee fanden sie kein Mikroplastik, obwohl die Tiefsee als Senke für Marine Litter angesehen werden kann, besonders für dichte Kunststoffe wie PA, PVC und PET. Die Besiedelung von
Organismen, sogenanntes „biofouling“, kann bewirken, dass schwimmende oder schwebende Mikroplastik-Partikel zum Lebensraum für Algen und kleine Organismen werden, deshalb in ihrem Aggregat an Dichte zunehmen und dabei zu Boden sinken. Die Kontamination von Sedimenten des Schwarzen Meeres sind vergleichbar $\mathrm{zu}$ anderen europäischen Regionen, jedoch wird beispielsweise die Menge von Marine Litter im Schwarzen Meer doppelt so hoch eingeschätzt, als jene im Mittelmeer (Slobodnik et al. 2018).

Über die Wege und Transportvorgänge ist wenig bekannt, Wind- und Wassertransport spielen jedenfalls eine bedeutende Rolle (Bertling et al. 2018b). Die Modellierung der Verbreitungswege von Quellen zu Lagern und Senken macht bisher in der Wissenschaft aufgrund der Datenlage und der Komplexität des Sachverhaltes noch Schwierigkeiten. Mit dieser Studie soll versucht werden die Verbreitungswege in einem Modell abzubilden.

\section{Zielsetzung der Studie}

Ziel ist die Identifizierung und Quantifizierung von Kunststoffemissionen, deren Quellen und Senken, sowie deren Transportwege (Transfer) in die Umwelt. Die Berechnung der Kunststoffeinträge in Oberflächengewässer steht dabei im Fokus, da schlussendlich die durch Flüsse exportierte Kunststoffmenge abgeschätzt werden soll. Der Fokus dieser Studie liegt auf den quantitativ relevantesten Kunststoff-Emissionen. Das Ziel soll mittels Durchführung einer Materialflussanalyse erreicht werden.

\section{Definitionen zentraler Begriffe}

Marine Littering Als Marine Litter werden alle Gegenstände und Materialien definiert, welche vom Menschen produziert sind und in die Weltmeere gelangen, wo sie schlussendlich verbleiben. Gegenwärtig gibt es keinen allgemeingültigen Ansatz zur Definition von Marine Litter. Hartmann et al. (2019) definiert den kunststoffhaltigen und weitaus größten Teil von Marine Litter ("plastic debris") als feste und bei $20^{\circ} \mathrm{C}$ unlösliche Objekte, welche synthetische oder stark modifizierte natürliche Polymere als essentielle Bestandteile haben. 
Littering Das Land Steiermark definiert dies folgendermaßen: „Littering bezeichnet das achtlose Wegwerfen von Abfällen an öffentlichen Plätzen und in der Natur." Das beinhaltet sowohl Zigarettenstummel, Kaugummi, im weitesten Sinn auch achtlos weggeworfene Autoreifen und Haushaltsgeräte (Land Steiermark A14). In diesem Bericht wird von Littering gesprochen, wenn ein Kunststoffgegenstand intentionell oder nicht intentionell in die Umwelt gelangt.

Makroplastik Kunststoffe, welche gröBer als $5 \mathrm{~mm}$ sind, werden als Makroplastik bezeichnet (Bertling et al. 2018b).

Mikroplastik Kunststoffe mit einer Größe zwischen $1 \mu \mathrm{m}$ und $5 \mathrm{~mm}$ werden als Mikroplastik bezeichnet, alle kleineren Partikel werden als Nanoplastik bezeichnet (Hartmann et al. 2019). Die AutorInnen unterscheiden als Hauptkriterien zur Beschreibung von Mikroplastik die Größe (i), die Form (ii), die Farbe (iii) und die Quelle (iv). In dieser Studie wird das Mikroplastik mit dem Nanoplastik zusammen in der Kategorie „Mikroplastik“ betrachtet, da Nanoplastik ein relativ neuer Begriff ist und keine vergleichbaren Quantifizierungen zu den Emissionen von Nanoplastik vorliegen. Mikroplastik wird weiter differenziert in primäres und sekundäres Mikroplastik.

Primäres Mikroplastik: Primäres Mikroplastik sind Kunststoffe, die in Form von kleinen Partikeln direkt in die Umwelt freigesetzt werden. Sie können ein Additiv zu Produkten wie Waschmittel, Toilettenartikel und Kosmetika (z. B. Duschgel) sein. Es kann auch durch den Abrieb von Makroplastik-Gegenständen während der Herstellung, des Gebrauchs oder der Wartung entstehen (Boucher und Friot 2017).

Primäres Mikroplastik - Typ A \& B: Primärer Mikroplastik Typ A wird von der Industrie in Form von synthetischen Granulaten hergestellt und ist somit intentionell erzeugtes Mikroplastik. Rohstoffe für die weitere Verwendung heißen Pellets (Bertling et al. 2018b). Eine andere Form sind Mikrokügelchen, die in industriellen Sandstrahlern, oder sogenannte "Microbeads“, die in Reinigungspasten oder kosmetischen Produkten als Reinigungspellets verwendet werden. Bei der Herstellung, Verladung und dem Transport von Kunststoffpellets treten regelmäßig Emissionen auf (Hohenblum et al. 2015). Durch Abrieb beim Gebrauch von Kunststoffgegen- ständen entsteht Primäres Mikroplastik Typ B (Bertling et al. 2018b).

Sekundäres Mikroplastik: Sekundäres Mikroplastik entsteht durch verschiedene physikalische oder chemische Alterungs- und Abbauprozesse aus Kunststoffprodukten (Bertling et al. 2018b). Die Fragmentierung in kleinere Teilchen passiert dabei in unterschiedlichem Ausmaß, abhängig von gewissen Umweltfaktoren.

\section{Methodik}

Die Materialflussanalyse (MFA) ist eine systematische Berechnung von ,stocks“ and "flows" (Cencic und Rechberger 2008). Der Gegenstand dieser Studie ist nicht nur ein Stoff, sondern die Kunststoff-Emissionen im Allgemeinen (also mehrere Stoffe: PP, PE, PA, etc.). Die MFA beinhaltet folgende Elemente:

- Fluxes [t/a] stellen den Transport eines Materials von einem Prozess zum nächsten dar und verbinden die Prozesse. Fluxes, welche in das System führen, werden als Importe bezeichnet. Fluxes, welche das System verlassen, werden als Exporte bezeichnet.

- Prozesse sind Orte, an denen die Transformation, der Transport oder die Speicherung bestimmt wird (Cencic und Rechberger 2008). Hier fließen Emissionen zusammen oder werden aufgeteilt. Die Prozesse sind mit Transferquotienten ausgestattet, welche determinieren, welcher Anteil eines oder mehrerer EingangsFluxes in die jeweiligen AusgangsFluxes münden. Normalerweise werden Prozesse als „black box" mit bekannten oder berechneten Transferquotienten betrachtet, Prozesse können aber auch als Subsystem modelliert werden (Cencic und Rechberger 2008).

- Als Quellen werden Prozesse bezeichnet, an denen KunststoffEmissionen entstehen.

- Als Lager werden Prozesse bezeichnet, bei denen das Material eine Zeit lang verweilt, bevor es schlussendlich weiter transportiert wird.

- Als Senken werden Prozesse bezeichnet, an denen das Material entweder für immer bleibt oder zerstört wird.

\subsection{Systemdefinitionen}

Das untersuchte Material in der Materialflussanalyse ist primäres Mikroplastik und Makroplastik/Littering. Sekundäres Mikroplastik kann aufgrund der Komplexität der Zerfalls- und Transferprozesse im Umfang dieser Arbeit nicht modelliert werden. Das System beinhaltet die Modellierung der Emissionsquellen und -senken von Mikro- und Makro-Kunststoffen in der Steiermark, sowie den Transfer zwischen diesen Elementen und den Export in das darunterliegende Flusssystem bis ins Schwarze Meer. Quantifiziert wird in Tonnen pro Jahr [t/a] oder Gramm pro Kopf und Jahr [g/cap*a].

Für die Berechnung der KunststoffEmissionen der Steiermark werden nur jene Emissionsquellen betrachtet, bei denen sich mindestens zwei wissenschaftliche Analogien (vergleichbare Studien) zu anderen (europäischen) Regionen ziehen lassen und deren erwartete Emissionen (Durchschnittswerte anderer AutorInnen) $40 \mathrm{~g} / \mathrm{cap}^{* a}$ übersteigen.

\subsection{Generelle Annahmen}

\subsubsection{Annahme 1 - Gleichmäßige Pro- Kopf-Verteilung der Emissionen}

Die Emissionen der Haushalte und des Verkehrs sind im Mittel gleichmäßig über die ansässige Bevölkerung verteilt.

Erklärung: Das bedeutet, dass in ländlichen Gebieten pro Fläche weniger Emissionen anfallen, da dort die Bevölkerungsdichte geringer ist.

\subsubsection{Annahme 2 - Vergleichbarkeit zwischen Regionen}

Die Emissionen von Staaten mit ähnlichem wirtschaftlichen und gesellschaftlichen Hintergrund, sowie ähnlichen Abfallwirtschaftssystemen sind in ihrer Größe vergleichbar.

Begründung: Emissionen von Mikroplastik und Nanoplastik hängen stark mit der Größe der Bevölkerung, dem Wohlstand, der Anwesenheit und Effizienz der Abfallwirtschaft zusammen (Nizzetto et al. 2016) und in dicht besiedelten Gebieten wird mehr emittiert, wobei eine signifikante Beziehung zwischen der Bevölkerungsdichte und der Menge der Emission vorliegt (Browne et al. 2012). 


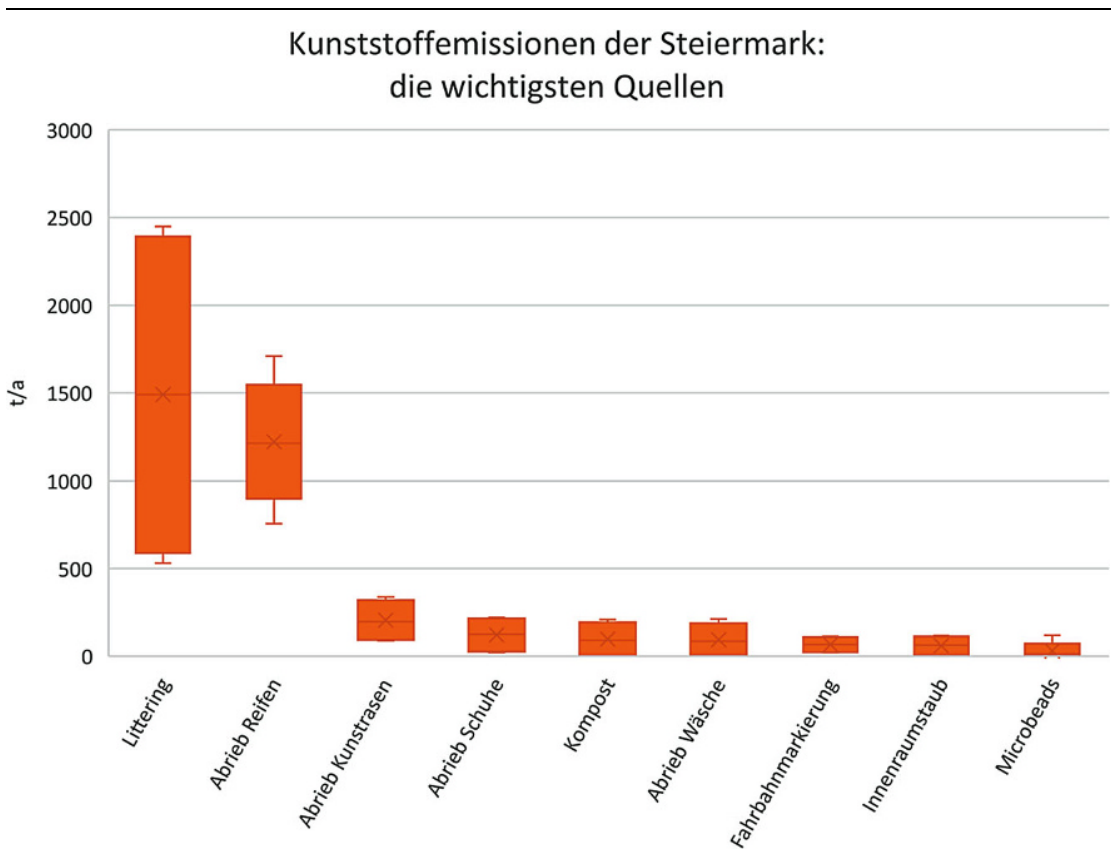

Abb. 1 Die wichtigsten Quellen von Kunststoff-Emissionen der Steiermark. (Eigene Darstellung)

\section{Ergebnisse}

Die Kunststoff-Emissionen der Steiermark insgesamt können nun mit einer hohen Wahrscheinlichkeit auf einige tausend Tonnen pro Jahr eingeschätzt werden. Die Emissionen von primärem Mikroplastik können mengenmäßig einigermaßen genau quantifiziert werden (siehe Abb. 1), während die Emissionen von Makroplastik schwieriger zu quan-

\section{Emissionsszenarien}

6000

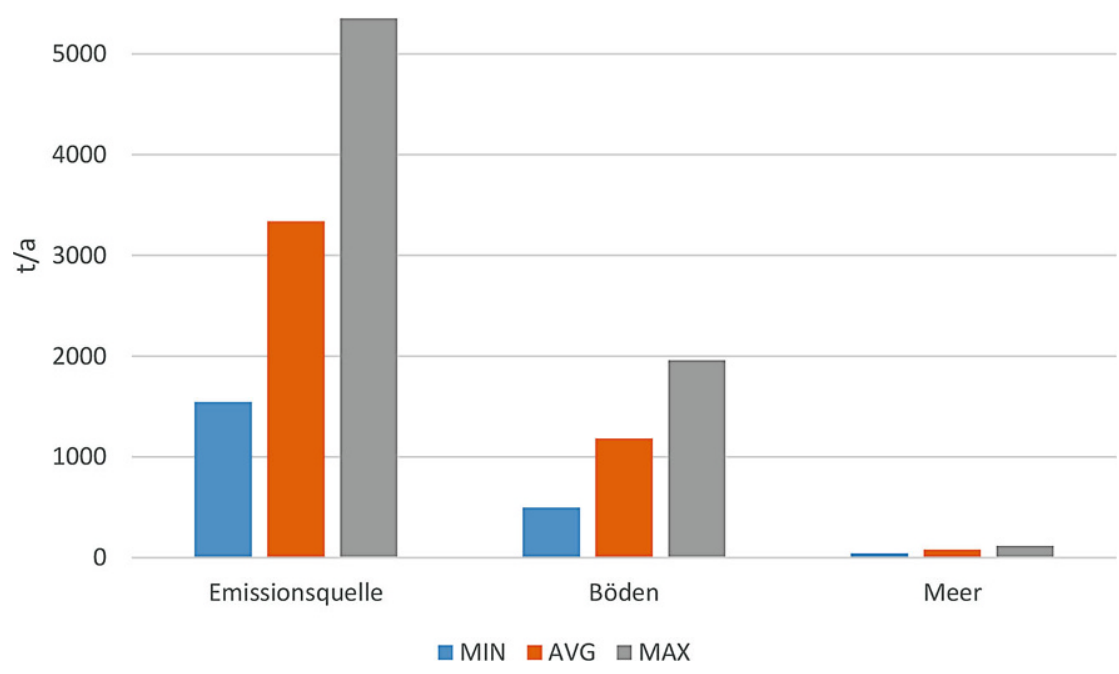

Abb. 2 Szenarien der Kunststoffemissionen der Steiermark
$2747 \pm 1444 \mathrm{t} / \mathrm{a}$. Im Modell werden $1848 \mathrm{t} / \mathrm{a}$ oder ca. $2 / 3$ jenes Mittelwertes kalkuliert. Die Emissionen durch Makroplastik Littering und Reifenabrieb bilden mit Abstand die größten Quellen im Modell. Die Menge des freigesetzten sekundären Mikroplastiks aus degradierendem Makroplastik in der Umwelt konnte aufgrund der Datenlage nicht modelliert werden.

Szenarien (MIN, AVG, MAX) Da klar geworden ist, dass die Modellierung von Kunststoffemissionen Unsicherheiten unterworfen ist, werden in Abb. 2 Szenarien (MIN, AVG und MAX) vorgestellt, unter der Beibehaltung der im Transfermodell festgelegten Transferraten. In der Grafik ist mit „Emissionsquellen" die Summe aller modellierten primären Emissionen gemeint, welche an den jeweiligen Emissionsquellen frei werden. Mit „Böden“ sind jene Mengen gemeint, welche im Boden verbleiben (Senke), und mit "Meer“ jene Mengen, welche in das Schwarze Meer gelangen. Die Emissionsmengen der jeweiligen Quellen und Senken ergeben zwischen Szenario MIN und MAX jeweils einen Unterschied des Faktors 3-4 (1545-5353t/a). Diese Zahlen sind jedoch nicht vollständig, da nicht $100 \%$ aller Emissionsquellen modelliert wurden. Es kann jedoch davon ausgegangen werden, dass die mengenmäßig relevantesten Quellen in der Studie berücksichtigt wurden.

\subsection{Transfermodell}

Mikro- und Makroplastik gelangen vom Ort der Emissionen über unterschiedliche Pfade in Oberflächengewässer und anschließend ins Meer und tragen so zum Marine Littering bei (siehe Abb. 3).

Die Sammlung durch die Straßenmeisterei, Sammelaktionen, Kläranlagen, Böden und Kraftwerksrechen sind relevante Barrieren für die Verbreitung von Kunststoffen hin zu Flüssen.

Als punktuelle Quellen werden die Emissionen im häuslichen Abwasser betrachtet: Abrieb Wäsche (synthetische Fasern), Innenraumstäube und Microbeads. Sie gelangen als Schmutzwasser vor allem im urbanen Bereich zum (großen) Teil in Mischwasserkanäle und im ländlichen Bereich hauptsächlich in Schmutzwasserkanäle. Die punktuellen Quellen tragen mit knapp $5 \%$ in etwa $155 \mathrm{t} / \mathrm{a}$ und somit einen relativ geringen Anteil zu den gesamten Emissionen bei. Sie sind von den 


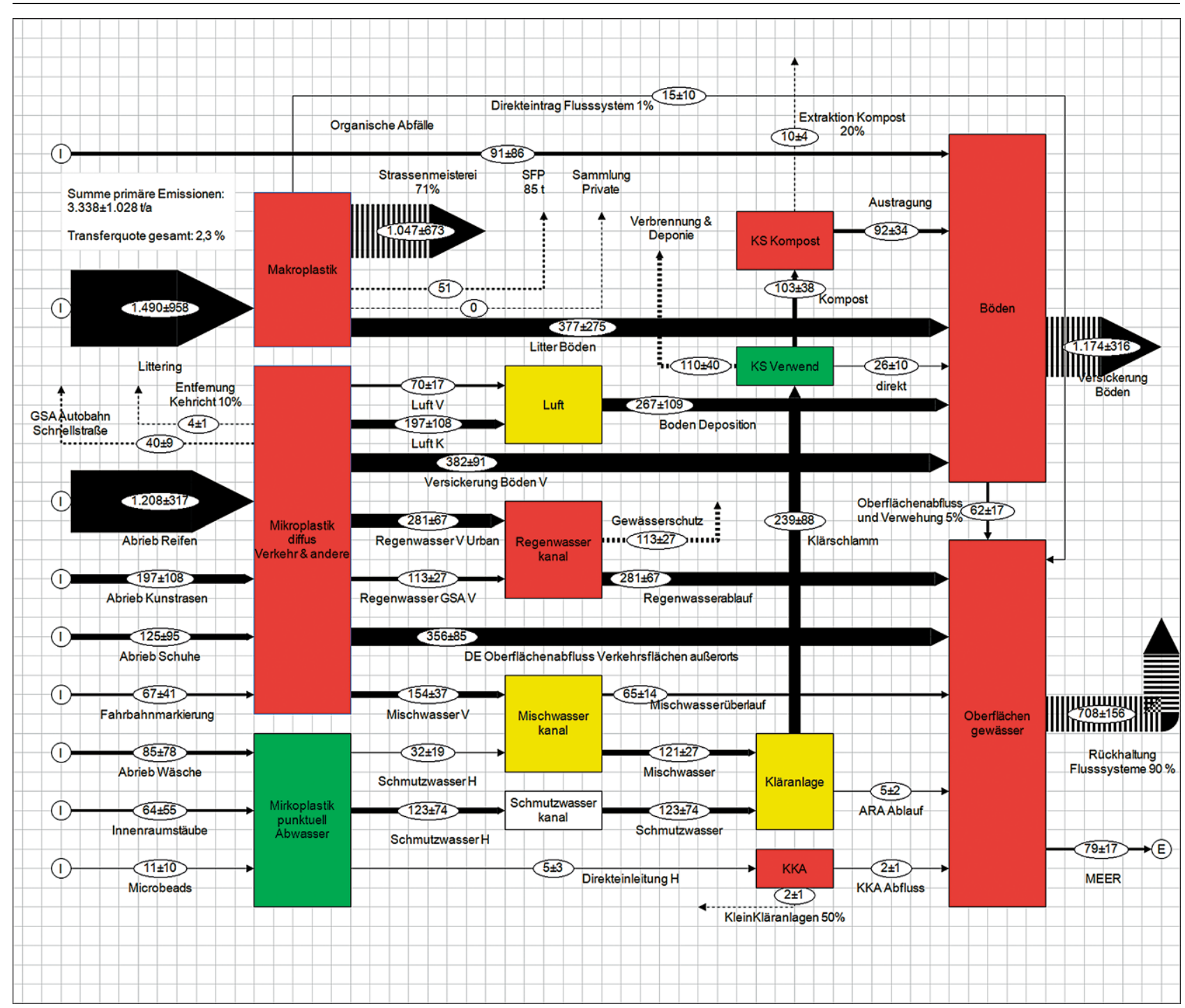

Abb. 3 Transfermodell. Screenshot aus STAN. (Eigene Darstellung). Die Farben geben eine Auskunft über die Einschätzung des Autors zur Güte der Daten: grün = gut, gelb = mittel, rot = schwach

punktuellen Einträgen in Böden oder ins Flusssystem zu unterscheiden.

Als diffuse Quellen von Mikroplastik werden jene Quellen betrachtet, deren Emissionen flächig auftreten. Dabei spielt der Ort der Emission eine große Rolle. Die relevantesten Flächen sind Verkehrsflächen, landwirtschaftliche Flächen und anderen Böden.

Auf Verkehrsflächen entsteht der diffuse Abrieb von Reifen, Fahrbahnmarkierung, Schuhsohlen, Kunstrasen und anderen Sportplätzen und einer Reihe kleinerer und nicht quantifizierter Emissionen. Ein Teil dieser Emissionen gelangt in die Luft (Annahme $5 \%$ vom Reifenabrieb und Fahrbahnmarkierung basierend auf [Verschoor et al. 2016]), ein größerer Teil (Annahme $95 \%$ ) bleibt auf den Verkehrsflä- chen oder straßennahen Böden, wovon, vor allem im urbanen Raum, ein Teil des Mikroplastiks (Annahme 4t) durch die Kehrdienste entsorgt wird (Land Steiermark A14). Die Emissionen auf Verkehrsflächen gelangen im urbanen Raum in die Mischwasserkanalisation und Regenwasserkanalisation im ländlichen Raum, sowie auf Autobahnen und Schnellstraßen hauptsächlich in straßennahe Böden und über die Regenwasserkanalisation oder direkt vom Straßenabfluss in das Flusssystem (Clara et al. 2014). Die Durchschnittswerte der Emissionen auf Verkehrsflächen bilden mit 1400 t/a ungefähr $42 \%$ der gesamten modellierten Emissionen einen bedeutenden Anteil im Modell. Der absolute Großteil davon (35\%) fällt auf den Reifenabrieb.
Das siedlungswasserwirtschaftliche Kanalsystem der Steiermark besteht in Laufmetern bemessen zu $90 \%$ aus Schmutzwasserkanälen (Trennsystem) und $\mathrm{zu} 10 \%$ (Clara et al. 2014) aus Mischwasserkanälen (Mischsysteme). Die Steiermark hat damit im Vergleich zu den anderen Bundesländern Österreichs einen sehr kleinen Anteil des Mischsystems, das jedoch für die Rückhaltung von Mikroplastik als das deutlich effektivere angesehen wird. In Kläranlagen wird das ankommende Schmutzwasser aus Mischwasserkanälen und Schmutzwasserkanälen aufgefangen, gereinigt und in den Vorfluter eingeleitet.

Mischwasserkanäle erfahren regelmäßig Mischwasserentlastungen, wobei Mikroplastik in unbehandeltem 
Abwasser hauptsächlich im urbanen Bereich in das Flusssystem gelangt $(65 \pm 14 \mathrm{t} / \mathrm{a})$. Der Weiterleitungsgrad hin zu den Kläranlagen ist jener Wert, der erreicht werden sollte und beträgt in etwa $70 \%$ (Gruber Günter, Persönliche Kommunikation, Mard 18, 2020) der Abwassermenge. Eine Abwasseremissionsverordnung gibt es in Österreich jedoch bisher nicht. Regenwasserkanäle führen nicht zu Kläranlagen und erfahren daher eine Reinigung höchstens durch Gewässerschutzanlagen (GSA), wie z. B. Sedimentationsbecken.

Die Kläranlage bildet die wichtigste (und oft einzige) punktuelle Rückhalteeinheit für Mikroplastik im Schmutzwasser von fast allen häuslichen Abwässern (Wäsche, Innenraumstäube, Microbeads) und teilweise von industriellen Abwässern, sowie von Teilen der Abwässer von Verkehrsflächen (Abrieb Reifen, Fahrbahnmarkierung, Schuhsohlen). Europäische Kläranlagen weisen Rückhalteraten für Mikroplastik von meist deutlich über $90 \%$ auf. Im Modell wird von den in der Literatur gerne zitierten $98 \%$ ausgegangen (Ngo et al. 2019; Siegfried et al. 2017).

Auf landwirtschaftliche Flächen gelangen hingegen Mikroplastik-Emissionen im Kompost aus der Sammlung biogener Abfälle sowie des Grünschnitts und aus dem Klärschlamm. Das Transportverhalten des Mikroplastiks auf Böden ist kaum quantifiziert, jedoch versickert wahrscheinlich ein Teil mit der Zeit im Boden.

Makroplastik (Littering) wird vermutlich zum größeren Teil auf und in der Nähe von Verkehrsflächen, im weitesten Sinne auch Wanderwege etc., durch Littering freigesetzt. Ein kleiner Teil davon (Annahme 1\%) gelangt nach dem Modell auch direkt ins Flusssystem, ein kleiner Teil in die Kanalisation (hier nicht modelliert, da angenommen wird, dass diese Menge vernachlässigbar ist). Der Großteil jedoch kann zumindest potenziell noch an Land gesammelt und entsorgt werden. Dies geschieht auch in einem gewissen Maße im Rahmen von Flurreinigungen, wobei die Straßenmeisterei wahrscheinlich den größten Anteil an Litter sammelt; Annahme: $71 \%$ des gelitterten Makroplastiks (Bertling et al. 2018a). Im Rahmen des steirischen Frühjahrsputzes könnten ebenso ca. 51t/a (Land Steiermark A14 2019) gesammelt werden. Außerdem wird angenommen, dass auch private Sammlungen eine (kleine) Rolle spielen. Kunststoffe, wel- che nicht im Rahmen der Flurreinigung gesammelt werden, verbleiben in der Umwelt und sind den degradationsrelevanten Umwelteinflüssen ausgesetzt. Somit bilden sie eine potenzielle Quelle für sekundäres Mikroplastik. Ein Teil des Makroplastiks kann auch über Oberflächenabflüsse ins Flusssystem gelangen. Wie die Rückhalteraten der Flusssysteme bezüglich Makroplastik zu bewerten sind, lässt sich derzeit nicht beantworten. Die Rechenanlagen der Wasserkraftwerke zum Schutz der Turbine vor großen Gegenständen spielen dabei wahrscheinlich eine zentrale Rolle. Makroplastik (Littering) macht im Modell einen Anteil von knapp 45\% oder $1490 \mathrm{t}$ aus.

Böden sind nach dem Modell Lager und Senken zugleich für Mikro- und Makroplastik, da Kunststoffe teilweise versickern und eventuell degradieren, und teilweise wieder abgeschwemmt oder verweht werden. Der Abrieb von Reifen und Fahrbahnmarkierung trägt am größten zur Verschmutzung der Böden durch Kunststoffe bei. Gefolgt von gelittertem Makroplastik ( $377 \pm 275 \mathrm{t} / \mathrm{a}$ ) und Verwehungen $(267 \pm t / a)$ von Verkehrsflächen, Kunstrasenplätzen etc., der Austragung von unbehandeltem Klärschlamm (26 $\pm 10 \mathrm{t} / \mathrm{a})$ und kompostiertem Klärschlamm $(103 \pm 38 \mathrm{t} / \mathrm{a})$, sowie von kommunalen biogenen $\mathrm{Ab}$ fällen $(91 \pm 86 \mathrm{t} / \mathrm{a})$.

Dem Modell zufolge wurde von den direkten Einträgen in das Flusssystem der, hauptsächlich aus Reifenabrieb bestehende, Oberflächenabfluss von Verkehrsflächen außerorts als sehr bedeutend bewertet $(356 \pm 85 \mathrm{t} / \mathrm{a})$, ebenso wie der urbane Regenwasserablauf $(281 \pm 67 \mathrm{t} / \mathrm{a})$ und der Mischwasserüberlauf $(65 \pm 14 \mathrm{t} / \mathrm{a})$. Der Ablauf der kommunalen Kläranlagen oder von Kleinkläranlagen wird mengenmäßig als sehr gering angesehen $(5 \pm 2 \mathrm{t} / \mathrm{a}$ bzw. $2 \pm 1 \mathrm{t} / \mathrm{a}$ ), allerdings könnten sehr viele kleine und leichte Partikel, vor allem über die Kläranlage, frei werden.

Die Transferrate von Emissionsquellen hin zur finalen Senke, dem Schwarzen Meer, wurde mit 2,3\% berechnet. Die Sensitivitätsanalyse ergab, dass die Transferraten von Kunststoffen auf Böden und in Flüssen einen sehr großen Einfluss auf die Menge der Emissionen haben.

\section{Diskussion}

\subsection{Modellierung \& Datenlage}

Bei der Modellierung sind im Laufe des Prozesses teilweise Schwierigkeiten bezüglich der Datenverfügbarkeit und Zuverlässigkeit aufgetreten, wodurch Lücken identifiziert wurden. Das Transfermodell steht auf einer sehr schwachen Datenbasis, wodurch man nur gewisse Tendenzen darüber abschätzen kann, welche Größenordnungen von Kunststoff-Emissionen über welche Pfade transportiert werden. Bertling et al. (2018b) meinen Folgendes: „Globale Transferraten in Flüsse und Meere lassen sich aufgrund einer Vielzahl an sequenziellen und parallelen Transportvorgängen heute noch nicht modellieren" (Bertling et al. 2018b). Diese Aussage kann im Grunde nachvollzogen werden, da die Modellierung von Kunststoffemissionen aus der Steiermark ins Meer nur sehr bedingt mit Daten untermauerbar ist.

\subsection{Interpretation der Ergebnisse}

Es scheint schlüssig hinter den abweichenden Ergebnissen der AutorInnen bezüglich der jährlichen Menge einzelner Emissionsquellen von primärem Mikroplastik nicht nur Unterschiede in wahren Emissionen zu sehen, sondern auch unterschiedliche Annahmen, Methodiken und Vollkommenheit der Analysen. Dennoch sind die Ergebnisse der AutorInnen relativ in derselben Größenordnung, so dass davon ausgegangen werden kann, dass die Emissionen einzelner Quellen vergleichbarer Länder in etwa gleich hoch sind. Daher wird die Wahrscheinlichkeit als hoch angenommen, dass sich auch steirische Emissionen pro Kopf in derselben Größenordnung bewegen. Die Ergebnisse der Emissionsquellen werden daher als einigermaßen valide angesehen.

Die in der Literatur stark unterschiedlich geschätzten Transferraten, beispielsweise für Microbeads (von , $<10 \%$ “ bis , $50 \%$ “) verdeutlichen auch die Schwierigkeit in der Modellierung des Transfers allgemein. Die unterschiedlichen Schätzungen könnten auf unterschiedlichen Methodiken oder Annahmen basieren, oder auf unterschiedlichen Effizienzgraden europäischer Kläranlagen. Allerdings wird in diesem Bericht angenommen, dass die Effizienzgrade der Kläranlagen verschiedener Länder Nord- und West-Eu- 
ropas einigermaßen vergleichbar sein dürften.

Im Emissionsmodell ist der Reifenabrieb deutlich am größten und es besteht die Möglichkeit, dass größere Anteile als die modellierten von Verkehrsflächen in die Böden statt Gewässer gelangen. Das Waschen synthetischer Kleidung fungiert als größte Emissionsquelle häuslicher Abwässer. Allerdings ist das Verhalten dieser Fasern in der Kläranlage noch nicht klar.

Die berechnete Menge der Kunststoff-Emissionen von Microbeads ist insofern eine Frage der Definition des Stoffes, weil in dieser Studie nur konventionelles Mikroplastik in fester Form betrachtet wurde. In einer anderen Studie (Bertling et al. 2018b) wird geschätzt, dass die Menge an gelösten Polymeren, welche in das häusliche Abwasser gelangt, in etwa 26-mal größer sein könnte, als jene von (festem) Mikroplastik. Das entspräche hochgerechnet auf die Steiermark in etwa $285 \mathrm{t} / \mathrm{a}$, womit Microbeads die drittgrößte Emissionsquelle von Kunststoffen wäre.

Die Austragung von Kompost und Klärschlamm stellt eine Besonderheit in der Modellierung von Mikroplastikemissionen dar, weil sie sekundäre und keine primären Emissionen bilden. Das bedeutet einerseits, dass sämtlicher Kunststoff in Kompost und Klärschlamm bereits emittiert worden ist, bevor sie in das jeweilige Medium gelangen. So bilden Kompost und Klärschlamm einen Verbreitungsweg für Kunststoffe in die Umwelt und sind dann wiederum als diffuse Quelle zu betrachten.

Die Konzentrationen in behandelten Abwässern (Bläsing und Amelung 2018) unterscheiden sich stark. Das kann verschiedene denkbare Gründe haben:

- Unterschiede in tatsächlichen Emissionsfrachten (lokale \& temporale Varianz),

- Unterschiede der Kläranlagen bezüglich der Rückhalteraten bei der Reinigung,

- Unterschiede der Messtechniken (Maschenweite Sieb).

Im Rahmen dieses Modells ist es erstmals gelungen, eine Einschätzung der Mikroplastik-Konzentrationen in steirischem Klärschlamm zu geben: $239 \pm$ 88t/a Mikroplastik auf ca. 22.000t/a Klärschlamm (theoretische Konzentration in etwa $1,5 \% \mathrm{TS}$ ).
In der Steiermark fallen jährlich 95 Mio. $\mathrm{m}^{3} / \mathrm{a}$ Schmutzwasser (Clara et al. 2014) an, welche von Kläranlagen gereinigt und in den Vorfluter eingeleitet werden. Im Transfermodell emittiert der ARA-Ablauf $5 \pm 2$ t/a an Kunststoffen ausschließlich Mikroplastik. Die Konzentration des gereinigten Ablaufes steirischer Kläranlagen würde dementsprechend $0,05 \mathrm{~g} / \mathrm{m}^{3}$ betragen.

Das Ergebnis der Emissionsquellen kann insgesamt als einigermaßen valide betrachtet werden. Die Emissionsszenarien in den Fällen MIN und MAX unterscheiden sich um einen Faktor von 3,4 .

Die Vergleichbarkeit der Emissionsquellen über Landesgrenzen hinweg ist relativ hoch, während die Transferraten in der Literatur teils sehr unterschiedlich sind und küstennahe Staaten auch nicht gut mit der Steiermark vergleichbar sind. Beispielsweise wird davon ausgegangen, dass der Abrieb von Autoreifen in den nord- und zentraleuropäischen Ländern pro Person einigermaßen gleich ist, während im Vergleich zu diesen Ländern die topographischen Eigenschaften der Steiermark und der damit verbundene Transfer in vielen Aspekten sehr unterschiedlich sind. Beispiel: Alle Länder zu denen Analogien gezogen wurden haben Meerzugang. Außerdem kann nicht von einer vollkommenen Vergleichbarkeit der Effizienz der Kanalsysteme und Kläranlagen ausgegangen werden. Deshalb sollten die Transferraten mit großer Vorsicht interpretiert werden.

Im Rahmen dieser Arbeit ist klar geworden, dass das Konzept von „Quellen, Lager und Senken“ nur bedingt auf inerte Stoffe, wie Kunststoffe anwendbar ist. Denn gerade auf und in Böden ist unklar was mit den Kunststoffen passiert, ob sie degradiert werden oder welcher Teil durch die Witterung ins Flusssystem gelangt. Böden stellen also gewissermaßen Lager und Senken zugleich dar.

Im Vergleich $\mathrm{zu}$ den von Jambeck et al. (2015) geschätzten 4,8-12,7 Mio. Tonnen an Kunststoffen, welche pro Jahr ins Meer geraten, trägt die Steiermark in etwa $0,0001 \%(0,00034$ $0,00099 \%$ ) bei, bei einem globalen Bevölkerungsanteil von rund $0,0167 \%$. Somit emittiert die Steiermark mit 64 g/cap*a (35-88) relativ wenig, im Vergleich zum weltweiten Durchschnitt nach Jambeck et al. (2015) (623$1649 \mathrm{~g} /$ cap*a). Im Verhältnis emittiert ein Mensch im globalen Durchschnitt danach 16-mal (7-48) so viele Kunststoffe ins Meer, wie ein/e durchschnittliche/r Einwohner/in der Steiermark. Das erscheint insofern nachvollziehbar, weil die Steiermark ein Binnenland ohne Meerzugang ist, und aus der Steiermark quasi nur über Flüsse Kunststoffe ins Meer emittiert werden, und nicht über eine direkte Küstenlinie. Andererseits funktionieren die Abfallwirtschaftssysteme in Nord- und Zentraleuropa (Analogien) im Vergleich zu vielen anderen Ländern der Erde relativ gut, was eine geringe Emissionsmenge erklärt.

\subsection{Zeitliche Varianz der Konzentrationen durch Überschwemmungen}

Je nach Wasserführung ergeben sich starke Differenzen zwischen Niederund Hochwasser: Durch schwere Überflutungen wurden $70 \%$ der in einer Studie (Hurley et al. 2018) betrachteten Mikroplastik-Verunreinigungen von Flusssedimenten weggespült. Die AutorInnen ziehen den Schluss, dass bei Überflutungen Mikroplastik effektiv flussabwärts transportiert wird. Die Frage kommt auf, inwiefern die Ergebnisse einer Konzentrationsmessung hochgerechnet werden können. Strände und Flussufer können als Lager betrachtet werden.

\subsection{Relevanz für die Abfallwirtschaft}

Littering ist im Vergleich $\mathrm{zu}$ anderen Umweltproblemen eine sehr sichtbare Form der Emission. Marine Littering und generell die Vermeidung von Kunststoffabfällen erfahren dadurch große Aufmerksamkeit in der Gesellschaft, vor allem aber in der Wissenschaft, der Wirtschaft und der Politik. Die Gefahren von Mikroplastik für einzelne Lebewesen oder Ökosysteme im Meer sind schwierig einschätzbar, und die Verschmutzung von Litter am Murufer kann manchem auch als hauptsächlich kosmetisches Problem erscheinen. Die Bewertung der Frage der Relevanz für die Umwelt ist somit aus unserer Sicht noch nicht möglich. Ein Großteil der Kunststoff-Emissionen bleibt im Land, denn das Mikroplastik aus häuslichen Abwässern wird zu einem bedeutenden Teil auf den Böden ausgetragen und der Reifenabrieb versickert zum Teil in straßennahen Böden. Auch vom Makroplastik, von dem noch ein größerer Teil von der Straßenmeisterei 
entfernt wird, verbleiben wahrscheinlich große Mengen in der Natur, bis sie möglicherweise wieder von Reinigungsaktionen beseitigt und gesammelt werden, falls sie noch nicht begraben oder degradiert sind.

Mikroplastik kann vermutlich nur zu einem kleinen, aber signifikanten Teil als Problem der Abfallwirtschaft betrachtet werden, da die meisten Emissionen in der Nutzungsphase von Produkten (Autoreifen, Wäsche, etc.) meist durch Abrieb entstehen. Jedoch entsteht meistens durch die Verarbeitung von kommunalen biogenen Abfällen eine Mikroplastikemission und durch die Verwendung (mit oder ohne Kompostierungsprozess) von Klärschlamm ebenfalls. Diese beiden Prozesse müssen daher im Fokus der Abfallwirtschaft stehen, wenn es um die Vermeidung von Emissionen geht, die allerdings weniger relevant für die Meere, als mehr für die heimischen Böden sind. Daneben könnten heimische Kunststoffproduzenten oder Recyclingunternehmen gewisse für das Marine Littering relevante Emissionen von Mikro- und Makroplastik erzeugen, besonders dann, wenn ihre Fabriken nahe am Flusssystem sind.

\subsection{Fazit}

Littering ist mit $45 \%$ (von 3338t/a) der modellierten Kunststoff-Emissionen die größte Emissionsquelle der Steiermark und findet diffus im ganzen Land statt.

Die relevantesten Mikroplastik-Mengen entstehen auf Verkehrsflächen (Reifenabrieb $36 \%$, Abrieb Fahrbahnmarkierung 2\%, Abrieb Schuhe 4\%), bei Infrastrukturen (Abrieb Kunstrasen 6\%) und in privaten Haushalten (Abrieb Wäsche $3 \%$, Innenraumstäube $2 \%$, Microbeads $<1 \%$, bei Außerachtlassen der gelösten Polymere). Die Emissionen der Abfallwirtschaft sind im Vergleich dazu nachrangig und gelangen hauptsächlich über den Kompost (3\%) auf die steirischen Böden.

Böden sind komplexe Umweltkompartimente in Bezug auf den Transfer von Kunststoffen und die genauen Dy- namiken sind unbekannt. Sie können als Lager und Senken zugleich betrachtet werden, da Kunststoffe teils über den Oberflächenabfluss von Böden in Oberflächengewässer gelangen, teils im Boden versickern.

Ähnlich gestaltet sich die Situation bei Oberflächengewässern. Die Dichte und die Größe der Partikel spielen beim Transport eine große Rolle. Partikel können sedimentieren, oder teilweise wieder mobilisiert werden, und auch durch Überschwemmungen von Flüssen auf benachbarte Böden geraten. Im Modell wird angenommen, dass $10 \%$ der in Oberflächengewässer emittierten Kunststoffe das Meer erreichen.

Anthropogene Rückhaltemaßnahmen bilden relevante Senken für Mikroplastik (Kläranlage, Kompostierung) und Makroplastik (Flurreinigungen durch Straßenmeisterei, Steirischer Frühjahrsputz). Es wird davon ausgegangen, dass die Kläranlage ca. 98\% des Mikroplastiks und $100 \%$ des Makroplastiks zurückhält, während die Straßenmeisterei in etwa $71 \%$ des Makroplastiks sammeln könnte. Als finale Senke der Kunststoffe, welche über die Mur ins Meer gelangen, wird das Schwarze Meer betrachtet.

Im Vergleich mit anderen Abschätzungen zu Emissionen in Oberflächengewässer (Nizzetto et al. 2016) und das Meer (Siegfried et al. 2017) wurde der Beitrag der Steiermark als sehr hoch bzw. hoch modelliert. Im Vergleich $\mathrm{zu}$ den gemessenen Konzentrationen europäischer Flüsse und dem Durchfluss der Mur wurden für die Mur sehr viel höhere Emissionsmengen ins Meer modelliert. Dabei muss allerdings auch in Betracht gezogen werden, dass die Messung von Kunststoff-Konzentrationen immer einem Fehler unterliegen, da durch die Maschenweite von Sieben bei der Probenahme kleinere Partikel teilweise verloren gehen, wodurch bei Angaben zu Konzentrationen systematisch ein zu niedriger Wert angegeben wird. Außerdem könnte die Rolle von Hochwasser sehr bedeutend sein, was die Aussagekraft gemessener Konzentrationen bei Normalwasser relativieren könnte. Jedenfalls ist klar geworden, dass die Beiträge der Steiermark, aufgrund der nicht vorhandenen Küstenlinie zum Meer und einer funktionierenden Abfallwirtschaft, im Verhältnis $\mathrm{zu}$ anderen Regionen der Erde relativ gering sind. Im Vergleich $\mathrm{zu}$ den von Jambeck et al. (2015) berechneten Emissionen, welche vom Land ins Meer gelangen, emittiert ein Mensch der Steiermark mit 35-88 g/cap*a um in etwa das 16-fache (7-48) weniger als ein durchschnittlicher Mensch der Erde. Die Steiermark emittiert Mengen in der Größenordnung von ungefähr 0,0001 \% der weltweiten Kunststoff-Emissionen vom Land ins Meer.

Funding Open access funding provided by Montanuniversität Leoben.

Open Access Dieser Artikel wird unter der Creative Commons Namensnennung 4.0 International Lizenz veröffentlicht, welche die Nutzung, Vervielfältigung, Bearbeitung, Verbreitung und Wiedergabe in jeglichem Medium und Format erlaubt, sofern Sie den/die ursprünglichen Autor(en) und die Quelle ordnungsgemäß nennen, einen Link zur Creative Commons Lizenz beifügen und angeben, ob Änderungen vorgenommen wurden.

Die in diesem Artikel enthaltenen Bilder und sonstiges Drittmaterial unterliegen ebenfalls der genannten Creative Commons Lizenz, sofern sich aus der Abbildungslegende nichts anderes ergibt. Sofern das betreffende Material nicht unter der genannten Creative Commons Lizenz steht und die betreffende Handlung nicht nach gesetzlichen Vorschriften erlaubt ist, ist für die oben aufgeführten Weiterverwendungen des Materials die Einwilligung des jeweiligen Rechteinhabers einzuholen.

Weitere Details zur Lizenz entnehmen Sie bitte der Lizenzinformation auf http://creativecommons.org/licenses/ by/4.0/deed.de. 


\section{Verwendete Literatur}

Andrady, A. L. (2011): Microplastics in the marine environment. Marine Pollution Bulletin 62(8), 1596-1605. https://doi.org/10.1016/j. marpolbul.2011.05.030

Bertling, J., Hamann, L., \& Bertling, R. (2018a): Kunststoffe in der Umwelt: Mikro- und Makroplastik. https://doi.org/10.24406/UMSICHT-N497117

Bertling, J., Hamann, L., \& Hiebel, M. (2018b): Mikroplastik und synthethetische Polymere in Kosmetikprodukten sowie Wasch-, Putz- und Reinigungsmitteln. Fraunhofer Verlag. https:// doi.org/10.24406/UMSICHT-N-490773

Bläsing, M., \& Amelung, W. (2018): Plastics in soil: Analytical methods and possible sources. Science of the Total Environment, 612, 422-435. https://doi.org/10.1016/j.scitotenv.2017.08.086 Boucher, J., \& Friot, D. (2017): Primary microplastics in the oceans: A global evaluaton of sources. Gland: IUCN. Retrieved from https:// www.iucn.org/content/primary-microplastics oceans. Zugegriffen: 3.3.2020

Browne, M.A. [Mark Anthony], Crump, P., Niven, S. J., Teuten, E., Tonkin, A., Galloway, T., \& Thompson, R. (2012): Accumulation of Microplastic on Shorelines Woldwide: Sources and Sinks. Environmental Science \& Technology, 45(21), 9175-9179. https://doi.org/10.1021/ es201811s

Browne, M.A. [Mark A.], Dissanayake, A., Galloway, T. S., Lowe, D. M., \& Thompson, R. C. (2008): Ingested Microscopic Plastic Translocates to the Circulatory System of the Mussel Mytilus edulis (L.). Environmental Science \& Technology, 42(13), 5026-5031. https://doi.org/ $10.1021 /$ es800249a

Carpenter, E. J., Anderson, S. J., Harvey, G. R., Miklas, H. P., \& Peck, B. B. (1972): Polystyrene spherules in coastal waters. Science, 178(4062), 749-750. https://doi.org/10.1126/science.178. 4062.749

Cencic, \& Rechberger (2008): Material Flow Analysis with Software STAN. In A. Möller, B. Page, \& M. Schreiber (Eds.), Environmental informatics and industrial ecology: 22nd International Conference on Informatics for Environmental Protection ; enviroinfo 2008 ; proceedings of the 22nd International Conference Environmental Informatics-Informatics for Environmental Protection, Sustainable Development and Risk Management, September 10-12, 2008, Leuphana University Lueneburg, Germany. Aachen: Shaker.

Clara, M., Ertl, T., Giselbrecht, G., Gruber, G., Hofer, T., Humer, F., ..., Windhofer, G. (2014): Spurenstoffemissionen aus Siedlungsgebieten und von Verkehrsflächen. Wien. Bundesministerium für Land- und Forstwirtschaft, Umwelt und Wasserwirtschaft

Colton, J. B., JR, Burns, B. R., \& Knapp, F. D. (1974): Plastic particles in surface waters of the northwestern atlantic. Science, 185(4150), 491-497. https://doi.org/10.1126/science.185. 4150.491

Connors, K.A., Dyer, S. D., \& Belanger, S. E. (2017): Advancing the quality of environmenta microplastic research. Environmental Toxicology and Chemistry, 36(7), 1697-1703. https://doi. org/10.1002/etc.3829

Essel, R., Engel, L., Carus, M., \& Ahrens, R. H. (2015): Sources of microplastics relevant to marine protection in Germany. Hürth: Umweltbundesamt

European Parliament (2018): Plastic Oceans: MEPs back EU ban on polluting throwaway pla- stics by 2021: Press Release. Retrieved from https://www.europarl.europa.eu/news/en/ press-room/20181009IPR15501/plastic-oceansmeps-back-eu-ban-on-polluting-throwawayplastics-by-2021. Zugegriffen: 3.3.2020 Hartmann, N. B., Hüffer, T., Thompson, R. C. Hassellöv, M., Verschoor, A., Daugaard, A. E., ... Wagner, M. (2019): Are We Speaking the Same Language? Recommendations for a Definition and Categorization Framework for Plastic Debris. Environmental Science \& Technology, 53(3), 1039-1047. https://doi.org/10.1021/acs. est.8b05297

Hohenblum, P., Helmut Frischenschlager, Hubert Reisinger, Robert Konecny, Maria Uhl, Simone Mühlegger, ... Rolf Rindler (2015): Plastik in der Donau: Untersuchung zum Vorkommen von Kunststoffen in der Donau in Österreich; im Auftrag des BMLFUW und de Ämter der LR Oberösterreich, Niederösterreich und Wien. Report/Umweltbundesamt. Wien Umweltbundesamt.

Hurley, R., Woodward, J., \& Rothwell, J. J. (2018): Microplastic contamination of rive beds significantly reduced by catchment-wide flooding. Nature Geoscience, 11(4), 251-257. https://doi.org/10.1038/s41561-018-0080-1

Jambeck, J. R., Geyer, R., Wilcox, C., Siegler, T. R., Perryman, M., Andrady, A., ..., Law, K. L. (2015): Plastic waste inputs from land into the ocean. Science, 347(6223), 768. https://doi.org/ 10.1126/science. 1260352

Jang, M., Shim, W. J., Cho, Y., Han, G. M., Song, Y. K., \& Hong, S. H. (2020): A close relationship between microplastic contamination and coastal area use pattern. Water Research, 171, 115400. https://doi.org/10.1016/j.watres.2019. 115400

Jobling, S., Reynolds, T., White, R., Parker, M. G., \& Sumpter, J. P. (1995): A variety of environmentally persistent chemicals, including some phthalate plasticizers, are weakly estrogenic. Environmental Health Perspectives, 103(6) 582-587. https://doi.org/10.1289/ehp.95103582 Land Steiermark A14 (2019): Die Erfolgsbilanz: „Der Steirische Frühjahrsputz 2019“. Retrieved from https://www abfallwirtschaft.steiermark at/cms/beitrag/12736429/73033083/. Zugegriffen: 3.3 .2020

Lassen, C., Steffen Foss Hansen, Kerstin Magnusson, Fredrik Norén, Nanna Isabella Bloch Hartmann, Pernille Rehne Jensen, ... Anna Brinch (2015): Microplastics-Occurrence, effects and sources of releases to the environment in Denmark. Retrieved from https://backend. orbit.dtu.dk/ws/portalfiles/portal/118180844/ Lassen_et_al._2015.pdf. Zugegriffen: 3.3.2020 Liebmann, B. (2015): Mikroplastik in der Umwelt: Vorkommen, Nachweis und Handlungsbedarf. Report/Umweltbundesamt: REP-0550. Wien: Umweltbundesamt. Retrieved from http:// www.umweltbundesamt.at/aktuell/publikation en/publikationssuche/publikationsdetail/?pub_ id $=2141$

Liu, M., Lu, S., Song, Y. [Yang], Lei, L., Hu, J., Lv, W., ... He, D. (2018): Microplastic and mesoplastic pollution in farmland soils in suburbs of Shanghai, China. Environmental Pollution, 242 855-862. https://doi.org/10.1016/j.envpol.2018 07.051

Magnusson, Karin Eliasson, Anna Fråne, Kalle Haikonen, Johan Hultén, \& Mikael Olshammar, Johanna Stadmark, Anais Voisin (2017): Swedish sources and pathways for microplastics to the marine environment. Retrieved from https://www.ivl.se/webdav/files/Rapporter/ C183.pdf. Zugegriffen: 3.3.2020
Mani, T., \& Burkhardt-Holm, P. (2020): Seasonal microplastics variation in nival and pluvia stretches of the Rhine River-From the Swiss catchment towards the North Sea. Science of the Total Environment, 707, 135579. https://doi.org/ 10.1016/j.scitotenv.2019.135579

Ngo, P. L., Pramanik, B. K., Shah, K., \& Roychand, R. (2019): Pathway, classification and removal efficiency of microplastics in wastewate treatment plants. Environmental Pollution, 255 113326. https:// doi.org/10.1016/j.envpol.2019. 113326

Nizzetto, L., Futter, M., \& Langaas, S. (2016): Are Agricultural Soils Dumps for Microplastics of Urban Origin? Environmental Science \& Technology, 50(20), 10777-10779. https://doi.org/10. 1021/acs.est.6b04140

Rios, L. M., Moore, C., \& Jones, P. R. (2007): Persistent organic pollutants carried by synthetic polymers in the ocean environment. Marine Pollution Bulletin, 54(8), 1230-1237. https://doi. org/10.1016/j.marpolbul.2007.03.022

Ryan, P. G., Connell, A. D., \& Gardner, B. D. (1988): Plastic ingestion and PCBs in seabirds: Is there a relationship? Marine Pollution Bulletin, 19(4), 174-176. https://doi.org/10.1016/0025326X(88)90674-1

Siegfried, M., Koelmans, A. A., Besseling, E., \& Kroeze, C. (2017): Export of microplastics from land to sea. A modelling approach. Water Research, 127, 249-257. https://doi.org/10.1016/j. watres.2017.10.011

Slobodnik, J., Alexandrov, B., Komorin, V., Mikaelyan, A., Guchmanidze, A., \& M. Arabidze, A. K. (NOVEMBER 2018): National Pilot Monitoring Studies and Joint Open Sea Surveys in Georgia, Russian Federation and Ukraine, 2017: Draft Final Scientific Report. Retrieved from http:// emblasproject.org/wp-content/uploads/2019/ 07/EMBLAS-II_NPMS_JOSS_2017_ScReport_

FinDraft2.pdf. Zugegriffen: 3.3.2020

Sun, J, Dai, $X$, Wang, $Q$, van Loosdrecht, $M$. C.M., \& Ni, B.-J. (2019): Microplastics in wastewater treatment plants: Detection, occurrence and removal. Water Research, 152, 21-37. https://doi.org/10.1016/j.watres.2018.12.050

Sundt, Schulze, \& Syversen (2014): Sources of microplastic-pollution to the marine environment. Retrieved from https://www.miljodirekto ratet.no/globalassets/publikasjoner/M321/ M321.pdf. Zugegriffen: 3.3.2020

Teuten, E. L., Rowland, S. J., Galloway, T. S., \& Thompson, R. C. (2007): Potential for plastics to transport hydrophobic contaminants. Environmental Science \& Technology, 41(22), 7759-7764. https://doi.org/10.1021/es071737s

Thompson, R. C., Olsen, Y., Mitchell, R. P., Davis, A., Rowland, S. J., John, A. W. G., ... Russell, A. E. (2004): Lost at Sea: Where Is All the Plastic? Science, 304(5672), 838. https://doi.org/10. 1126/science.1094559

Turner, K. J., \& Sharpe, R. M. (1997): Environmental oestrogens-present understanding. Reviews of Reproduction, 2(2), 69-73. https:// doi. org/10.1530/ror.0.0020069

Verschoor A, de Poorter L, Dröge R, Kuenen J, \& de Valk E (2016): Emission of microplastics and potential mitigation measures : Abrasive cleaning agents, paints and tyre wear: Rijksinstituut voor Volksgezondheid en Milieu RIVM. National Institute for Public Health and the Environment Niederlande

World Economic Forum (2016): The New Plastics EconomyRethinking the future of plastics. Retrieved from http://www3.weforum. org/docs/WEF_The_New_Plastics_Economy. pdf. Zugegriffen: 3.3.2020 


\section{Weiterführende Literatur}

Land Steiermark A14. Abfall und Stoffflüsse 21. Umweltschutzbericht 2005/2006. Retrieved from https://www.umwelt.steiermark.at/cms/ dokumente/12567934_135843577/2601ae6e/
USB-05-06_LB-Luis-04-Abfall\%2BStoffl.pdf. Zugegriffen: 3.3.2020

Land Steiermark A14. Littering. Retrieved from https://www.abfallwirtschaft.steiermark.at/

cms/beitrag/12649239/73032309. Zugegriffen: 3.3.2020
Hinweis des Verlags Der Verlag bleibt in Hinblick auf geografische Zuordnungen und Gebietsbezeichnungen in veröffentlichten Karten und Institutsadressen neutral. 